\title{
Optimal Design and Analysis of the Stepped Core for Wireless Power Transfer Systems
}

\author{
Xiu Zhang ${ }^{1,2}$ and Xin Zhang ${ }^{1,2}$ \\ ${ }^{1}$ College of Electronic and Communication Engineering, Tianjin Normal University, Tianjin 300387, China \\ ${ }^{2}$ Tianjin Key Laboratory of Wireless Mobile Communications and Power Transmission, Tianjin Normal University, \\ Tianjin 300387, China
}

Correspondence should be addressed to Xin Zhang; ecemark@mail.tjnu.edu.cn

Received 21 September 2015; Accepted 14 January 2016

Academic Editor: Tariq S. Durrani

Copyright (C) $2016 \mathrm{X}$. Zhang and X. Zhang. This is an open access article distributed under the Creative Commons Attribution License, which permits unrestricted use, distribution, and reproduction in any medium, provided the original work is properly cited.

\begin{abstract}
The key of wireless power transfer technology rests on finding the most suitable means to improve the efficiency of the system. The wireless power transfer system applied in implantable medical devices can reduce the patients' physical and economic burden because it will achieve charging in vitro. For a deep brain stimulator, in this paper, the transmitter coil is designed and optimized. According to the previous research results, the coils with ferrite core can improve the performance of the wireless power transfer system. Compared with the normal ferrite core, the stepped core can produce more uniform magnetic flux density. In this paper, the finite element method (FEM) is used to analyze the system. The simulation results indicate that the core loss generated in the optimal stepped ferrite core can reduce about $10 \%$ compared with the normal ferrite core, and the efficiency of the wireless power transfer system can be increased significantly.
\end{abstract}

\section{Introduction}

Wireless power transfer technology has a history of more than one hundred years. Since the nineteenth century, Nikola Tesla proposed two coils which were named as Tesla coil to transfer the power between the two coils wirelessly [1]. Over the decades, after Tesla, the wireless power transfer system developed very slowly because of the limit of the electronic technology [2]. With the rapid development of modern technology in the new century, wireless power transfer system has great application potentials especially in implantable medical devices.

With the advent of power electronics, various implanted medical devices, such as deep brain stimulator, nerve stimulator, and cardiac pacemaker, are widely used in the medical field [3-5]. These devices can effectively prolong the patients' lives. In order to alleviate the patients' financial burden and their suffering, the technology of wireless power transfer has become popular in biomedical engineering.
Before the magnetic resonant coupling method is proposed, the wireless power transfer technology mainly has two types: one is far field method which focuses on microwave power transfer system; the other one is near field method which is applied in electrical toothbrush with very small distance. The far field method can transfer power over a long distance but the power transfer efficiency is very small. In addition, the systems using the far field mode need complicated detection devices to realize the power transfer from point to point. In the millimeter distance, the near field method can achieve high power transfer efficiency. When the power transfer distance is up to centimeters, the power transfer efficiency of near field systems is only about 1\% [6-8].

In 2007, the MIT research team published their findings in Science, and since then, the magnetic resonant coupling method has become the hottest methodology to realize wireless power transfer system. In order to distinguish from the above two methods, this method is named as midfield method. In this method, the power transfer distance can 


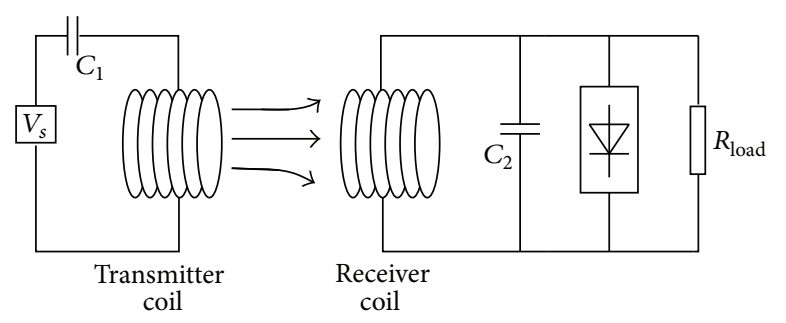

(a)

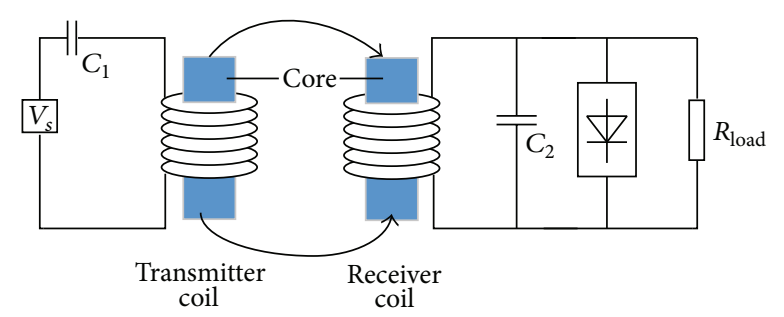

(b)

Figure 1: (a) Wireless power transfer system without a core. (b) Wireless power transfer system with a core.

be prolonged significantly with relative high power transfer efficiency $[9,10]$.

In this paper, the ferrite material is employed as the core to be applied into the wireless power transfer system. According to the application of the system, a new shape of the transmitter coil is proposed to improve the performance of the system. In addition, the stepped ferrite core is optimized to reduce the core loss and increase the efficiency of the wireless power transfer system.

\section{Structure of Wireless Power Transfer Systems}

Figure 1(a) presents the studied analyzing common wireless power transfer system. It consists of two coils with the same resonant frequency, and the coils are connected with compensated capacitors. In order to stretch the power transfer distance, ferrite cores are inserted into the two coils as shown in Figure 1(b). Previous research results [11] indicate that the receiver coil with the ferrite core can attain more magnetic flux linkages which are generated by the transmitter coil with the ferrite cores.

In this paper, the wireless power transfer system is applied to the deep brain stimulator which is implanted inside the skull of patients. Transmitter coil with a novel shape as shown in Figure 2 is proposed. In this system, the transmitter coil with a ferrite core has an arc along with the shape of the human head.

During the analysis, the four-layer concentric sphere head model [12-14] being used is modeled and simulated as shown in Figure 3. The four layers are the scalp, skull, cerebrospinal fluid (CSF), and brain. The properties of each layer in the human head model are listed in Table 1.

For deep brain stimulator, the receiver coil should be as small as possible. In order to obtain the maximum power transfer efficiency, the ferrite core inserted into the transmitter coil needs to be optimized. As shown in Figure 2, the angle $\theta$ represents the length of the ferrite core, while the angle $\alpha$ represents the relative position of the transmitter coil and the receiver coil. In addition, the angle $\varphi$ represents the chord of the transmitter coil. The specifications of the system are listed in Table 2.

In this paper, the resonant frequency of the coils is $3 \mathrm{MHz}$. The Nickel-zinc (NiZn) ferrite core is chosen as the core material which is inserted into the coils. NiZn ferrite has

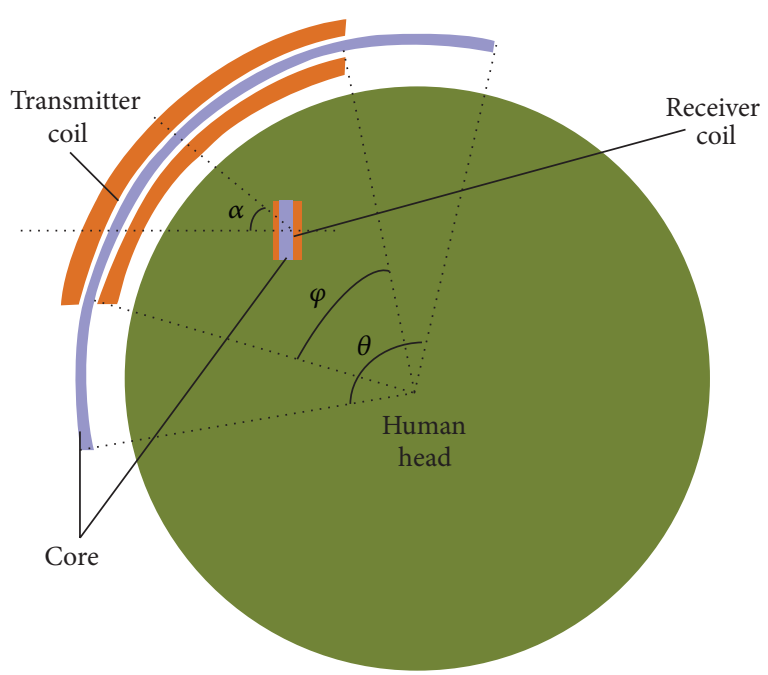

FIGURE 2: Novel shape of the wireless power transfer system with core applied to a deep brain stimulator.

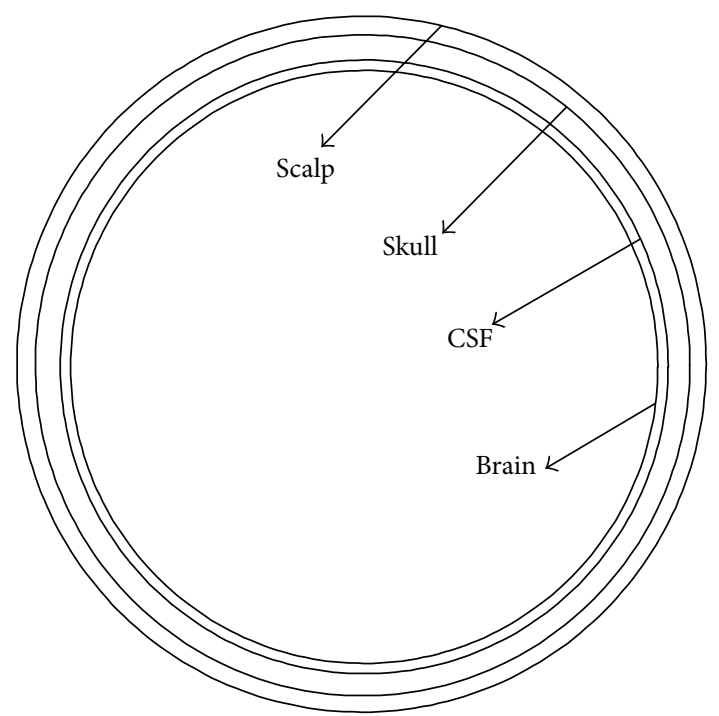

FIgURE 3: The four-layer concentric sphere head model.

higher resistivity which is more suitable for applications with operating frequencies above $1 \mathrm{MHz}$. 
TABLE 1: The properties of each layer in human head model.

\begin{tabular}{lcc}
\hline Tissue type & $\begin{array}{c}\sigma \\
\left(\Omega^{-1} \mathrm{~m}^{-1}\right)\end{array}$ & $\begin{array}{c}\text { Outer radius of } \\
\text { tissue type } \\
(\mathrm{mm})\end{array}$ \\
\hline Brain & 0.25 & 80 \\
CSF (cerebrospinal fluid) & 1.79 & 82 \\
Skull & 0.018 & 87 \\
Scalp & 0.44 & 92 \\
\hline
\end{tabular}

TABLE 2: Specifications of the wireless power transfer system.

\begin{tabular}{lc}
\hline Parameters & Values \\
\hline Angles & \\
$\alpha$ & $-40 \sim 40$ degrees \\
$\theta$ & $70 \sim 180$ degrees \\
$\varphi$ & 70 degrees \\
Diameter of wire & $1 \mathrm{~mm}$ \\
Diameter of coil & $4 \mathrm{~mm}$ \\
Length of receiver coil & $5 \mathrm{~mm}$ \\
Number of stranded wires & 70 \\
Turn number of transmitter coils & 10 \\
Turn number of receiver coils & 5 \\
Resonant frequency & $3 \mathrm{MHz}$ \\
Complementary capacitance in & $2.33 \mathrm{nF}$ \\
transmitter coil & \\
Complementary capacitance in receiver & $4.23 \mathrm{nF}$ \\
coil & \\
Saturation magnetic flux density of NiZn & $0.3 \sim 0.5 \mathrm{~T}$ \\
ferrite core &
\end{tabular}

\section{Methods and Analysis of the New Shape of the Transmitter Coil}

3.1. Finite Element Method. In order to reduce the $\mathrm{AC}$ resistance of the coils, the transmitter coil and the receiver coil are fabricated using Litz wires. According to the Maxwell equations, the two-dimensional (2D) magnetic field equation can be expressed as

$$
\nabla \cdot(\nu \nabla A)-\sigma \frac{\partial A}{\partial t}=-J_{s}
$$

where $A$ is the magnetic vector potential; $\nu$ and $\sigma$ are the reluctivity and the conductivity of the material, respectively; $J_{s}$ is the given current density.

The basic equations can be summarized as the following set of equations [15-20].

Field equation is as follows:

$$
\nabla \cdot(\nu \nabla A)-\sigma \frac{\partial A}{\partial t}+\frac{d_{p} N}{\operatorname{Sap}} i_{a d}+\frac{d_{p} N}{\operatorname{Sap}} i_{w}=-J_{s}
$$

Additional equation is as follows:

$$
-\frac{d_{p} N}{S a} \iint_{\Omega} \frac{\partial A}{\partial t} d \Omega+\frac{R_{d c}}{l} i_{a d}=0 .
$$

Electric circuit branch equation is as follows:

$$
-\frac{d_{p} N}{S a} \iint_{\Omega} \frac{\partial A}{\partial t} d \Omega-\frac{R_{d c}}{l} i_{w}=-\frac{1}{l} u_{w} .
$$

The induced voltage $V_{2}$ in the receiver coil is

$$
V_{2}=\omega \bar{B}_{2}(x) A_{2} N_{2},
$$

where $\omega$ is the angular frequency; $A_{2}$ is the cross section area of the receiver coil; $N_{2}$ is the number of turns of the receiver coil; $\bar{B}_{2}(x)$ is the average magnetic flux density in the receiver coil determined by

$$
\bar{B}_{2}(x)=\frac{1}{l_{r}} \int_{-l_{r} / 2}^{l_{r} / 2} B_{2}(x) d x,
$$

where $l_{r}$ is the length of the receiver coil. The power transfer efficiency of the system can be calculated by

$$
\eta=\frac{P_{2}}{P_{1}}=\frac{V_{2}^{2} / R_{2}}{I_{1}^{2} R_{1}}=\frac{\left(\omega \bar{B}_{2}(x) A_{2} N_{2}\right)^{2}}{I_{1}^{2} R_{1} R_{2}},
$$

where $P_{1}$ and $P_{2}$ are the input power in the transmitter coil and the output power induced in the receiver coil, respectively; $R_{1}$ and $R_{2}$ represent the resistance of the transmitter coil and the receiver coil, respectively; $I_{1}$ is the input current in the transmitter coil.

Magnetic flux lines in the wireless power transfer system are shown in Figure 4. It can be seen that the receiver coil can pick up the major bulk of the magnetic fluxes generated by the transmitter coil.

3.2. Analysis. The angle $\alpha$ represents the positional relationship between the transmitter coil and receiver coil. When the line connecting the centers of the transmitter coil and the receiver coil is parallel to $x$-axis, the value of angle $\alpha$ is equal to zero. The curve of power transfer efficiency varying with different $\alpha$ values is shown in Figure 5. It can be seen that the power transfer efficiency is maximum when the angle $\alpha$ is equal to -10 degrees.

The angle $\theta$ represents the length of the core inserted into the transmitter coil. The initial value of the angle $\theta$ is equal to 70 degrees when the lengths of the core and the transmitter coil are the same. The curve of power transfer efficiency varying with the different angle $\theta$ is shown in Figure 6. It can be seen that the power transfer efficiency is maximum when the angle $\theta$ is equal to 120 degrees.

\section{Analysis of the Stepped Core}

According to the theory of the electromagnetic field, if the thickness of the ferrite core is even, the magnetic flux density in the ferrite core is not uniform as shown in Figure 7. 


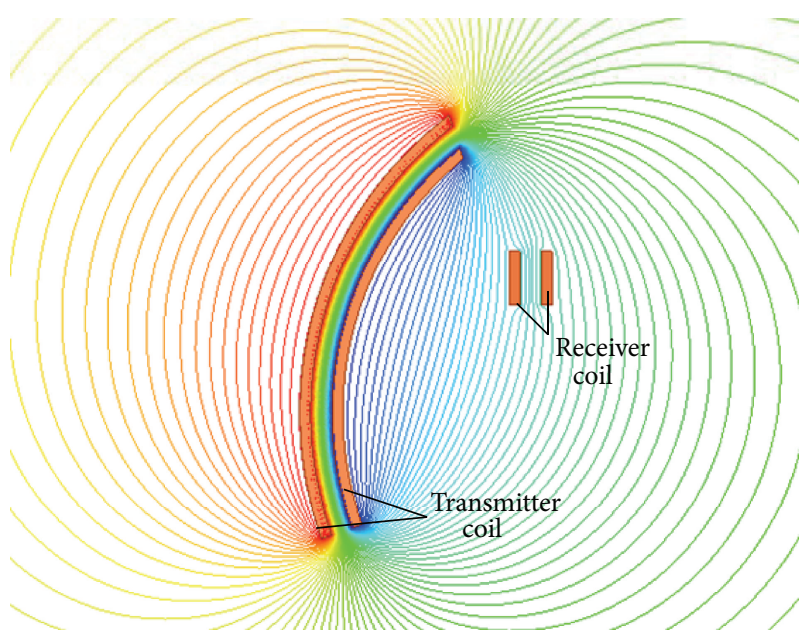

(a)

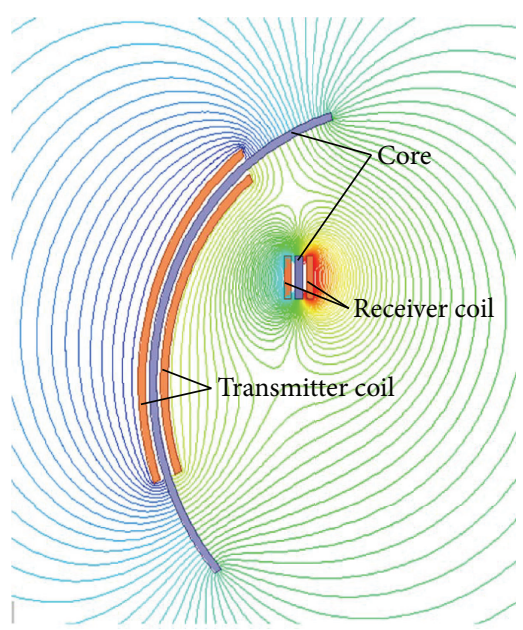

(b)

FIGURE 4: Simulation result of the magnetic flux lines of the proposed coil configuration: (a) without core; (b) with core.

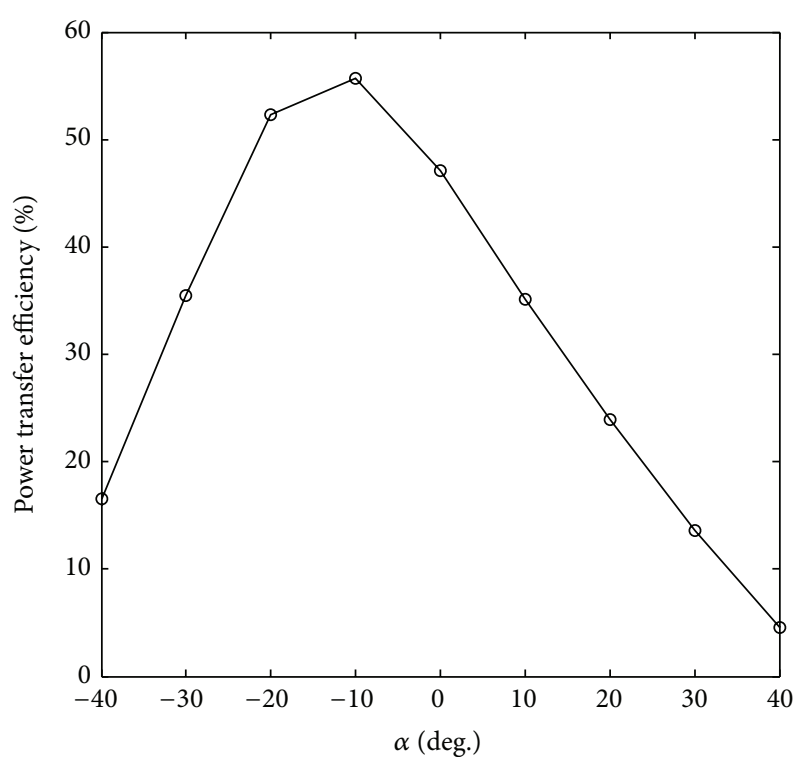

FIGURE 5: The efficiency of the system varies with $\alpha$.

The thickness of the ferrite core should be optimized to make the magnetic flux density uniform and reduce the amount of the ferrite material. The magnetic flux density in the ferrite core of the transmitter coil, as shown in Figure 7, can be numerically modeled as follows:

$$
\begin{aligned}
& B(\delta)=B_{0}-c \delta^{2} \quad|\delta|<\frac{\varphi}{2} \\
& B(\delta)=a\left(|\delta|-\frac{\varphi}{2}\right)+b \quad \frac{\varphi}{2}<|\delta|<\frac{\theta}{2} .
\end{aligned}
$$

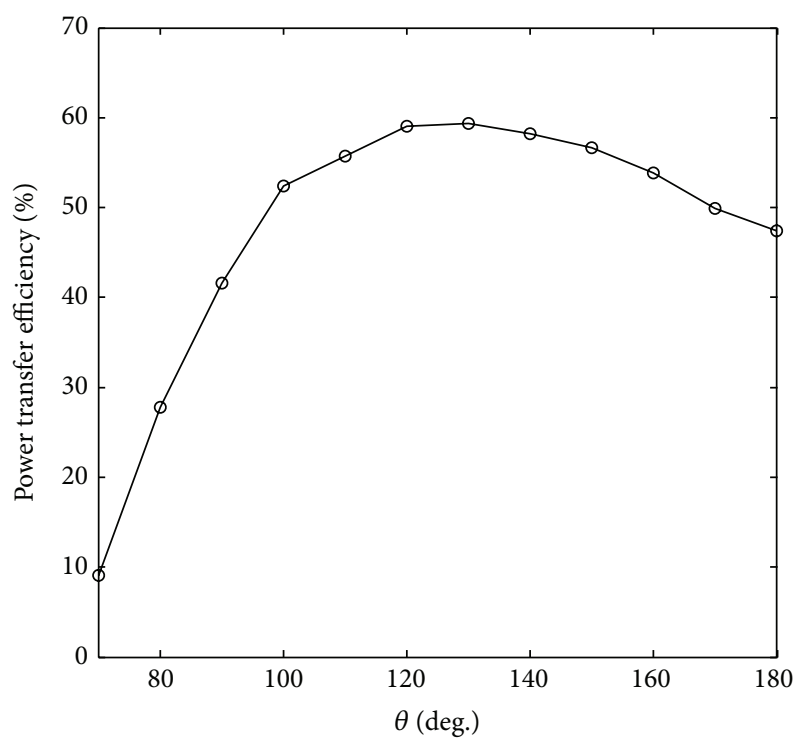

FIgURE 6: The efficiency of the system varies with $\theta$.

The parameters in the expressions are obtained by curve fitting from Figure 7 as follows:

$$
\begin{aligned}
B_{0} & =100.53, \\
c & =0.0276 ; \\
a & =-2.2053, \\
b & =67.1492 .
\end{aligned}
$$

According to the numerical model of Figure 7, the ferrite core can be divided into the wound section and the unwound section. In order to make the magnetic flux density uniform, the unwound section of the ferrite core will be fabricated as a stepped shape [11]. The magnetic flux density of the stepped core can be obtained by ( $8 \mathrm{~b})$. 


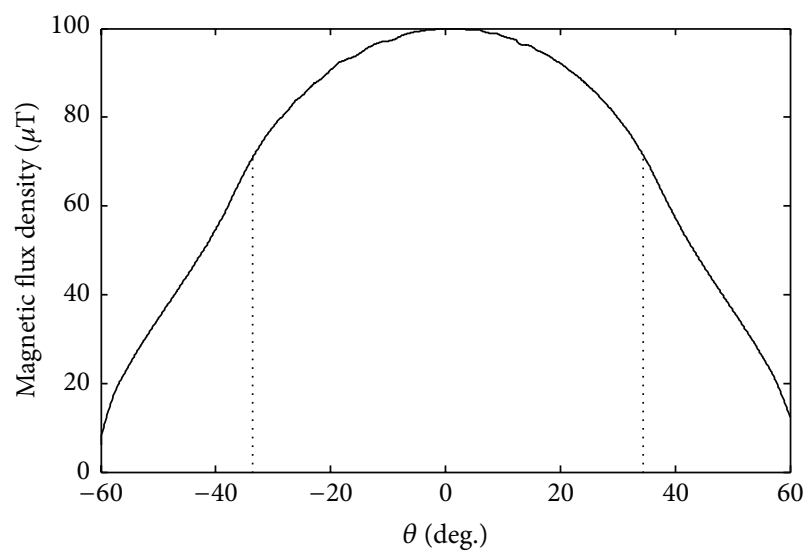

(a)

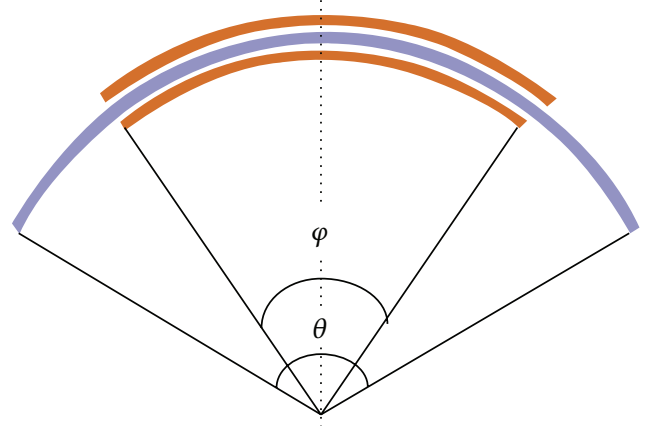

(b)

Figure 7: Simulated magnetic flux density for the transmitter coil with NiZn ferrite core. (a) Magnetic flux density in the ferrite core; (b) structure of the transmitter coil.

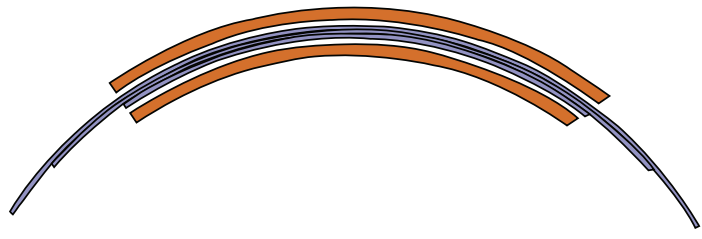

(a)

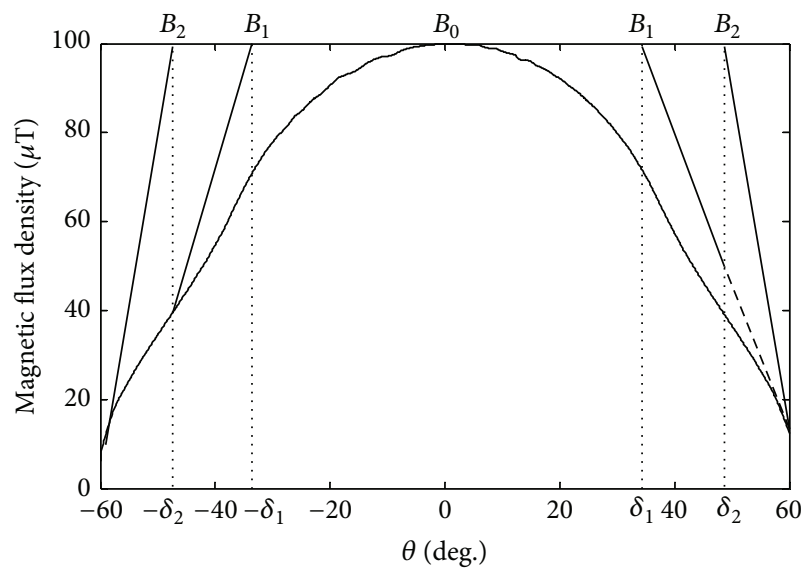

(b)

Figure 8: (a) Structure of the stepped core in the transmitter coil. (b) Assumed magnetic flux density in the stepped core.

As shown in Figure 8, the wound section is expressed as $B_{0}(\delta)$ which is the same as $(8 \mathrm{a})$. Each step of the unwound ferrite core is expressed as $B_{i}(\delta)(i=1,2, \ldots, n)$, where $n$ is the number of the total steps of the ferrite core. $B_{i}(\delta)$ can be described as

$$
\begin{aligned}
B_{i}\left(\delta_{i}\right) & =\max \left(B_{0}(\delta)\right) \\
B_{i}(\delta) & =a_{i}\left(|\delta|-x_{i}\right)+b_{i} .
\end{aligned}
$$

The magnetic flux at the stepped junction of the core should be continuous which agrees with the following description:

$$
B_{j+1} A_{j+1}=B_{j} A_{j}, \quad j=0,1,2, \ldots, n,
$$

where $A_{j}$ is the cross section area of the $j$ th step core. The magnetic flux density increases by the ratio of cross section area $A_{0} / A_{1}=n /(n-1)$. From (11), the relationship of the magnetic flux density between the stepped cores can be obtained such as

$$
B_{1}\left(\delta_{1}\right)=\frac{n}{n-1} B_{0}\left(\delta_{1}\right) .
$$

Then $\delta_{1}$ can be calculated as follows:

$$
\delta_{1}=\left(\frac{B_{0}}{n c}\right)^{1 / 2} .
$$

In this case, the unwound section of the ferrite core is divided into stepped core. $\delta_{1}$ is equal to $\varphi / 2$ which is 35 degrees from Table 2. In this condition, the number of step core $n$ should be 3 .

From Figure 8, the magnetic flux density is about $10 \mu \mathrm{T}$ when $\delta$ is equal to 60 degrees. Applying these values to (10b), $a_{1}$ can be determined and its value is about -3.6 . Similarly, $a_{2}$ can be calculated as -8.2 . Combined with (10a), (10b), and (11), $\delta_{2}$ can be determined by the following:

$$
\delta_{2}=\delta_{1}+\frac{B_{0}}{2 a_{1}} .
$$

Substituting the values of the coefficients into (14), $\delta_{2}$ is calculated as 49 degrees.

Based on the above calculated parameters, the comparison of the calculated result with the simulated result is shown 


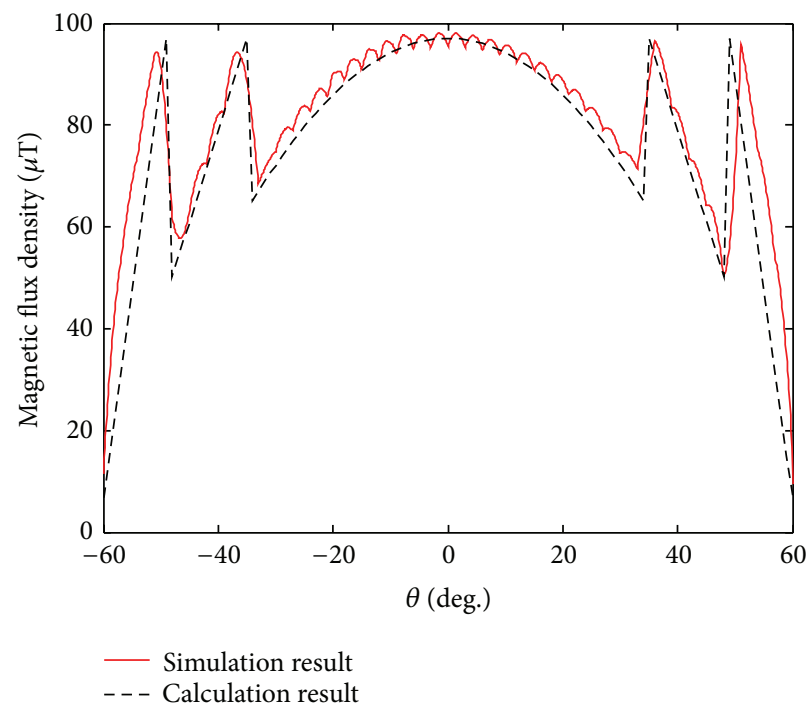

FIGURE 9: Comparison of the simulated magnetic flux density with the calculated result for the proposed stepped core.
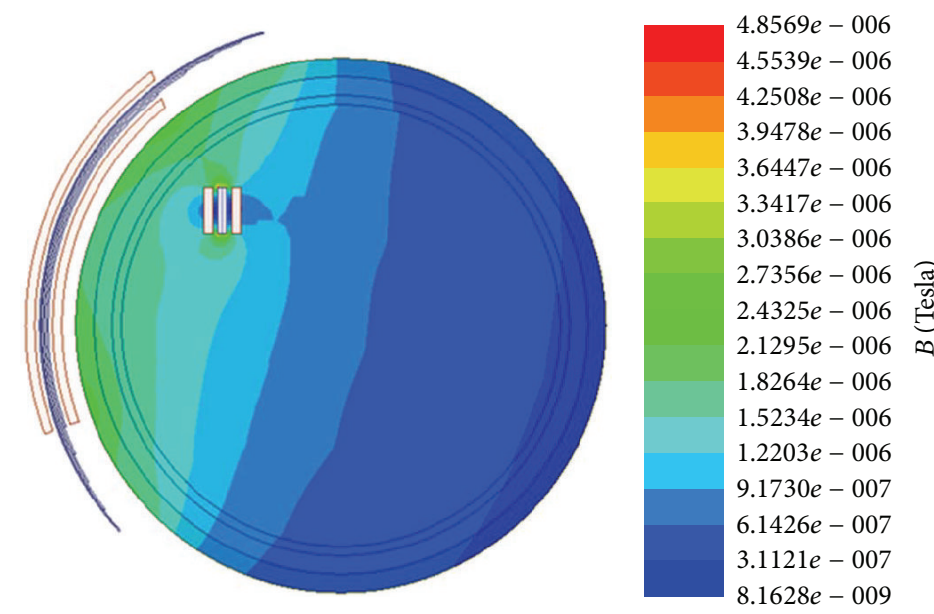

FIGURE 10: Distribution of the magnetic field strength in the human head.

in Figure 9. It indicates that the simulation result almost agrees with the calculation result.

As shown in Figure 4, the ferrite core can make the magnetic flux density concentrated, but the core loss generated in the ferrite core will weaken the performance of the wireless power transfer system. After simulation, the core loss in the stepped core will decrease about $20 \%$ compared with the normal core.

\section{Results}

Practically, the core loss in the ferrite material will increase the temperature of the coil; meanwhile, the higher temperature will decrease the saturation flux density of the ferrite material. The NiZn ferrite material used in this paper has a saturation flux density of about $400 \mathrm{mT}$ at room temperature. Considering the temperature caused by the core loss, the flux density in the core should not exceed $250 \mathrm{mT}$. For the deep brain stimulator, however, the security of the system to the patients is the most important problem that should be considered. According to the standard established by the Minister of Public Works and Government Services of Canada, the maximum magnetic field strength should not exceed $2.05 \mu \mathrm{T}$ [21].

In order to guarantee the security of the system, the distribution of the magnetic field strength in the human head is given in Figure 10. The curve of the magnetic field strength from left to right is shown in Figure 11. It can be seen that the maximum magnetic field strength in the human head is under $2 \mu \mathrm{T}$ which meets the above-mentioned standard. In this case, the input current in the transmitter coil is about $5 \mathrm{~mA}$. Under this excitation, the current induced in the receiver coil is shown in Figure 12. It indicates that the induced voltage in the receiver coil is above $3 \mathrm{~V}$ when the input current in the transmitter coil is about $5 \mathrm{~mA}$.

According to the above analysis, the wireless power transfer system will operate with the best performance when the angle $\alpha$ is equal to -10 degrees and the angle $\theta$ is equal 


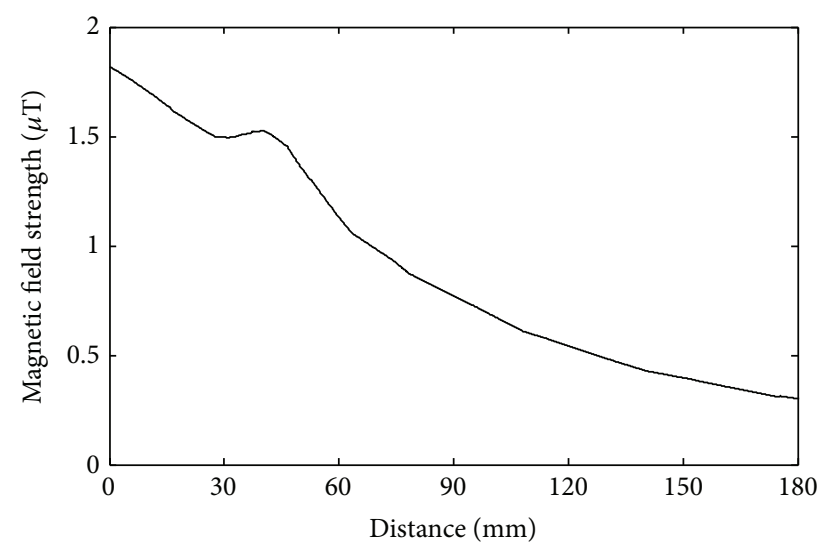

FIGURE 11: Curve of the magnetic field strength from left to right of the human head.

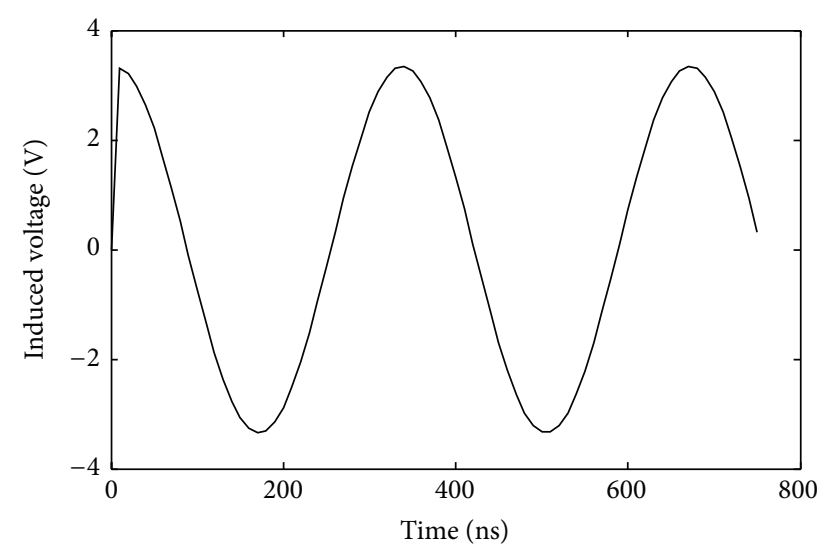

FIGURE 12: Induced voltage in the receiver coil when the input current in the transmitter coil is about $5 \mathrm{~mA}$.

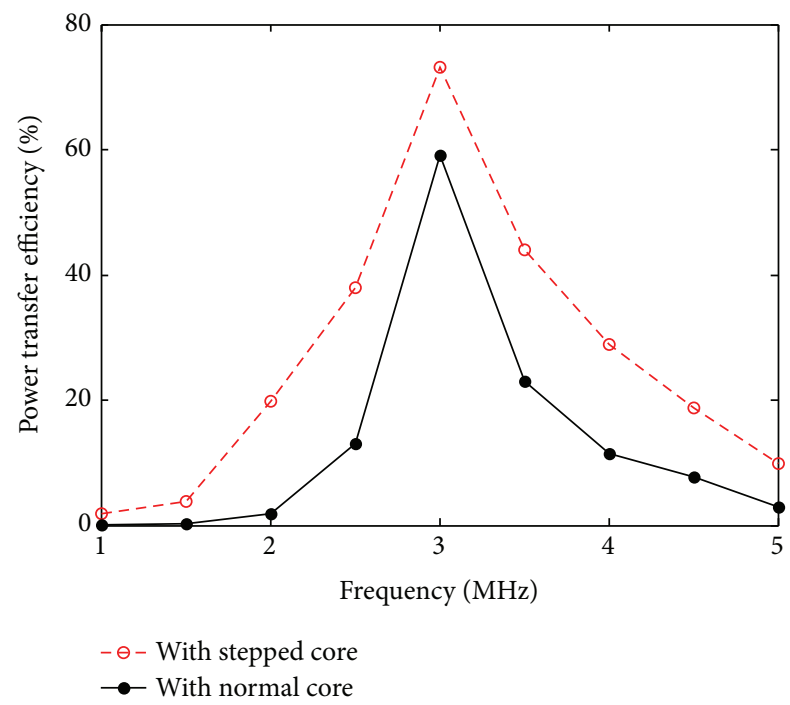

FIgURE 13: Power transfer efficiency with different frequencies. to 120 degrees. The power transfer efficiency of the system achieves the maximum value when the frequency of the excitation is $3 \mathrm{MHz}$ as shown in Figure 13. It can be seen that the power transfer efficiency of the system with the normal core is about $60 \%$, while the power transfer efficiency of the system with stepped core achieves larger than $70 \%$. The power transfer efficiency increases significantly. When the operating frequency deviates from the resonant frequency, the power transfer efficiency of the system will have a sharp decline.

\section{Conclusion}

In this paper, a transmitter coil with a novel shape is proposed for wireless power transfer system in implanted medical devices. A ferrite core which is suitable for high frequency application is inserted into both the transmitter coil and the receiver coil. The simulation results indicate that the receiver coil with the core can pick up more magnetic fluxes when compared with receive coils without the core. In addition, the relative positional relationship of the transmitter coil and the receiver coil and the length of the ferrite core inserted into the transmitter coil are both quantitatively analyzed. In order to reduce the core loss induced in the ferrite core, moreover, the normal core is replaced by the optimized stepped core. The simulation result indicates that this stepped core can reduce the core loss generated by the ferrite core and increase the power transfer efficiency of the wireless power transfer system significantly. The power transfer efficiency of the system can reach as high as $70 \%$. Such findings are instrumental for building a solid theoretical basis for future work in wireless power transfer systems.

\section{Conflict of Interests}

The authors declare that there is no conflict of interests regarding the publication of this paper.

\section{Acknowledgments}

This research was supported by the Applied Basic Research Program of Tianjin (15JCYBJC52300 and 15JCYBJC51500) and the Tianjin Thousand Youth Talents Plan Project of Tianjin Normal University (ZX110023 and ZX0471401505).

\section{References}

[1] R. Lomas, The Man Who Invented the Twentieth CenturyNikola Tesla-Forgotten Genius of Electricity, Headline Book Publishing, London, UK, 1999.

[2] W. C. Brown, "A survey of the elements of power transmission by microwave beam," IRE International Convention Record, vol. 9, no. 3, pp. 93-105, 1961.

[3] X. Liu, F. Zhang, S. A. Hackworth, R. J. Sclabassi, and M. Sun, "Wireless power transfer system design for implanted and worn devices," in Proceedings of the 35th Annual Northeast Bioengineering Conference, pp. 1-2, IEEE, Boston, Mass, USA, April 2009. 
[4] A. K. RamRakhyani, S. Mirabbasi, and M. Chiao, "Design and optimization of resonance-based efficient wireless power delivery systems for biomedical implants," IEEE Transactions on Biomedical Circuits and Systems, vol. 5, no. 1, pp. 48-63, 2011.

[5] Y. Yu, H. Hao, W. Wang, and L. Li, "Simulative and experimental research on wireless power transmission technique in implantable medical device," in Proceedings of the Annual International Conference of the IEEE Engineering in Medicine and Biology Society (EMBC '09), pp. 923-926, IEEE, Minneapolis, Minn, USA, September 2009.

[6] S. Y. Hui, "Planar wireless charging technology for portable electronic products and Qi," Proceedings of the IEEE, vol. 101, no. 6, pp. 1290-1301, 2013.

[7] S. Y. R. Hui, W. Zhong, and C. K. Lee, "A critical review of recent progress in mid-range wireless power transfer," IEEE Transactions on Power Electronics, vol. 29, no. 9, pp. 4500-4511, 2014.

[8] J. O. McSpadden and J. C. Mankins, "Space solar power programs and microwave wireless power transmission technology," IEEE Microwave Magazine, vol. 3, no. 4, pp. 46-57, 2002.

[9] A. P. Sample, D. J. Yeager, P. S. Powledge, A. V. Mamishev, and J. R. Smith, "Design of an RFID-based battery-free programmable sensing platform," IEEE Transactions on Instrumentation and Measurement, vol. 57, no. 11, pp. 2608-2615, 2008.

[10] A. Kurs, A. Karalis, R. Moffatt, J. D. Joannopoulos, P. Fisher, and M. Soljacic, "Wireless power transfer via strongly coupled magnetic resonances," Science, vol. 317, no. 5834, pp. 83-86, 2007.

[11] C. Park, S. Lee, G.-H. Cho, and C. T. Rim, "Innovative 5-moff-distance inductive power transfer systems with optimally shaped dipole coils," IEEE Transactions on Power Electronics, vol. 30, no. 2, pp. 817-827, 2015.

[12] S. Rush and D. A. Driscoll, "EEG electrode sensitivity-an application of reciprocity," IEEE Transactions on Biomedical Engineering, vol. 16, no. 1, pp. 15-22, 1969.

[13] T. F. Oostendorp, J. Delbeke, and D. F. Stegeman, "The conductivity of the human skull: results of in vivo and in vitro measurements," IEEE Transactions on Biomedical Engineering, vol. 47, no. 11, pp. 1487-1492, 2000.

[14] A. Liston, Models and image reconstruction in electrical impedance tomography of human brain function [Ph.D. thesis], Middlesex University, London, UK, 2003.

[15] J.-S. Zhao and W. C. Chew, "Integral equation solution of Maxwell's equations from zero frequency to microwave frequencies," IEEE Transactions on Antennas and Propagation, vol. 48, no. 10, pp. 1635-1645, 2000.

[16] J. W. Arthur, "An elementary view of Maxwell's displacement current," IEEE Antennas and Propagation Magazine, vol. 51, no. 6, pp. 58-68, 2009.

[17] W. N. Fu, P. Zhou, D. Lin, S. Stanton, and Z. J. Cendes, "Modeling of solid conductors in two-dimensional transient finite-element analysis and its application to electric machines," IEEE Transactions on Magnetics, vol. 40, no. 2, pp. 426-434, 2004.

[18] W. N. Fu and S. L. Ho, "Extension of the concept of windings in magnetic field electric circuit coupled finite element method," IEEE Transactions on Magnetics, vol. 46, no. 6, pp. 2119-2123, 2010.
[19] X. Zhang, S. L. Ho, and W. N. Fu, "Quantitative analysis of a wireless power transfer cell with planar spiral structures," IEEE Transactions on Magnetics, vol. 47, no. 10, pp. 3200-3203, 2011.

[20] X. Zhang, Y. Zhao, S. L. Ho, and W. N. Fu, "Analysis of wireless power transfer system based on 3-D finite-element method including displacement current," IEEE Transactions on Magnetics, vol. 48, no. 11, pp. 3692-3695, 2012.

[21] Limits of Human Exposure to Radiofrequency Electromagnetic Fields in the Frequency Range from $3 \mathrm{kHz}$ to $300 \mathrm{GHz}$, Minister of Public Works and Government Services, Canada, 1999. 

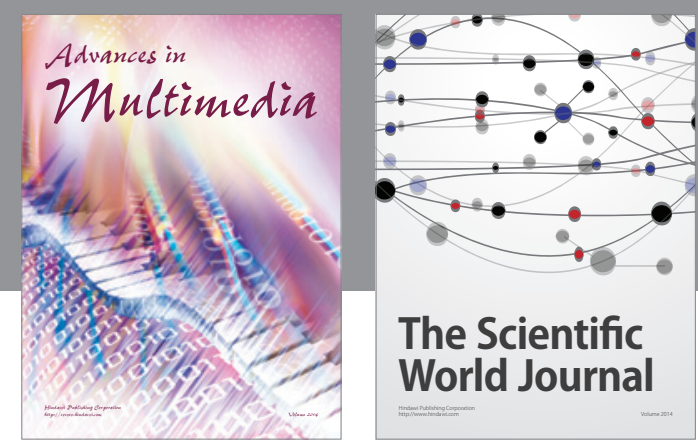

The Scientific World Journal
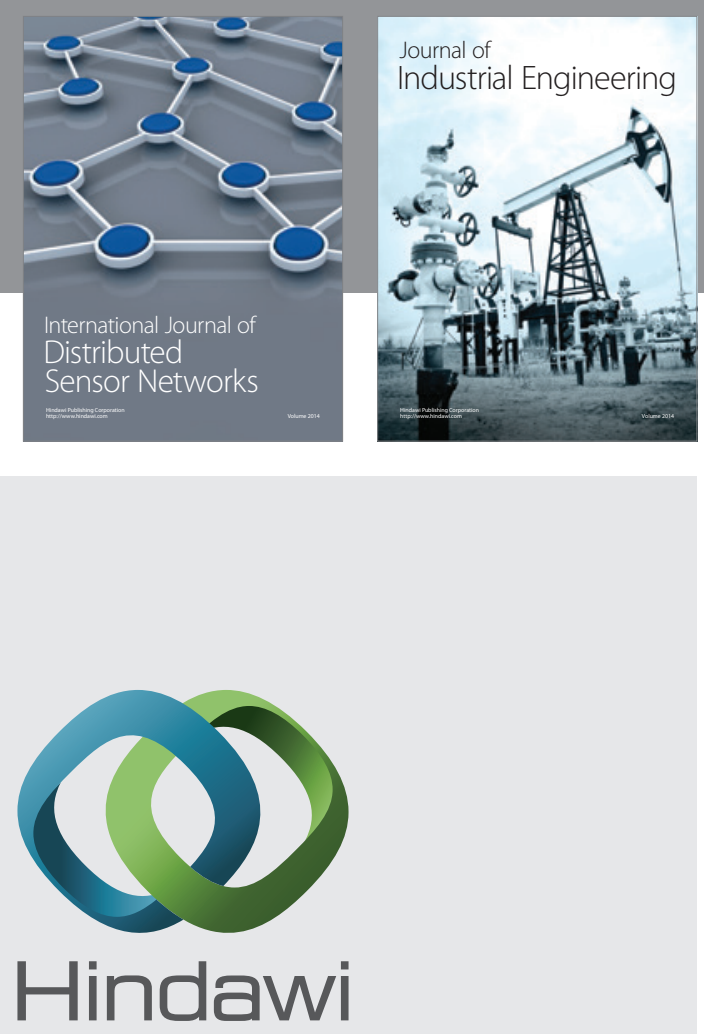

Submit your manuscripts at

http://www.hindawi.com

\section{Computer Networks} and Communications
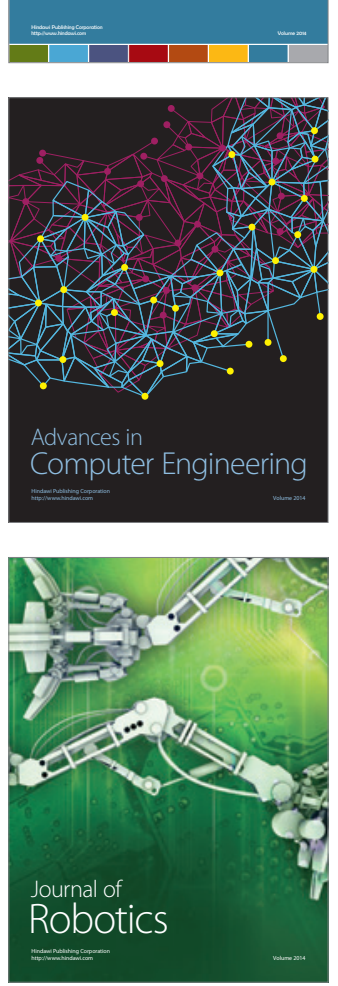
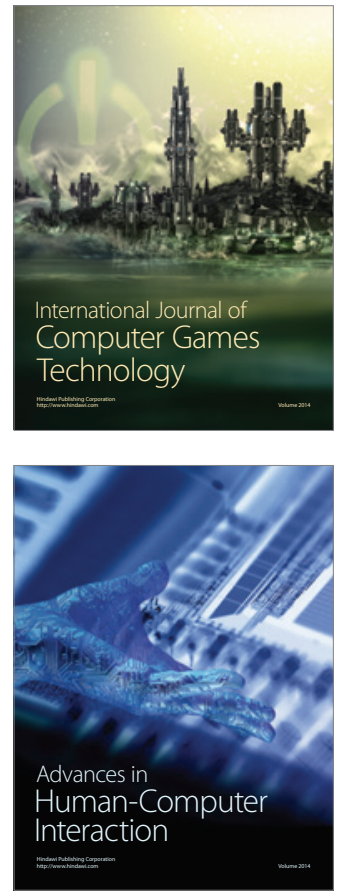
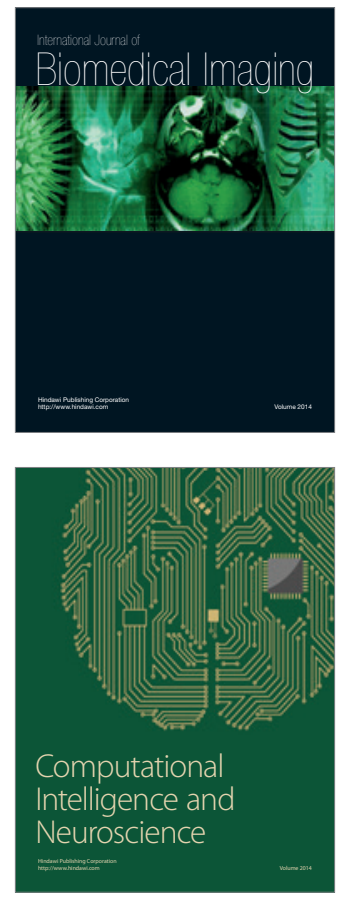
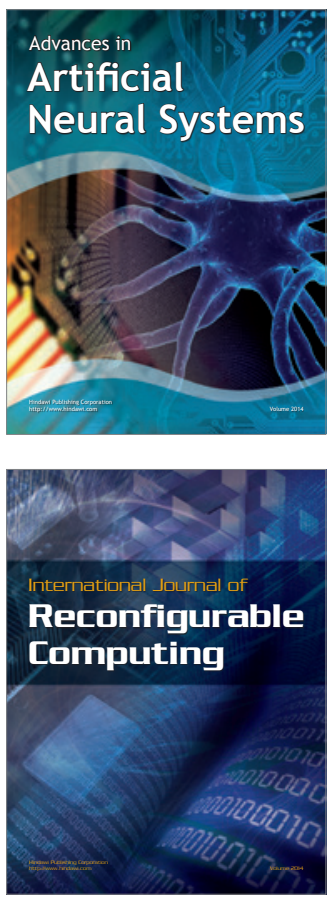
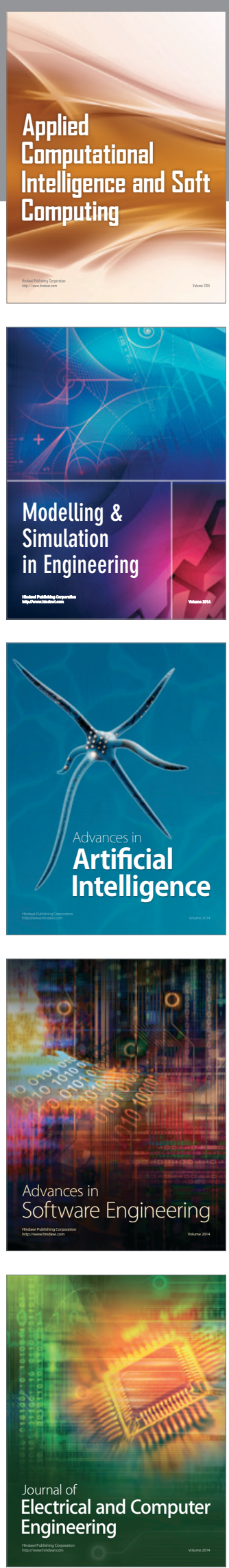